\title{
Kinderräume in der Coronakrise?
}

\section{Einführung}

Seit dem Ausbruch der Pandemie wird viel über Kinder berichtet und geforscht, sei es in den medialen und öffentlichen Debatten oder in wissenschaftlichen Studien. Sie stehen oftmals hinsichtlich der Übertragung des Virus, der Schließung von Kindertagesstätten und Schulen sowie der sozialen und psychischen Auswirkungen der Pandemie im Fokus. Jüngst wurde - nach einem Jahr mit der Pandemie und zwei intensiven Lockdowns - beispielsweise mit der „COPSY“-Studie (Januar 2021)[1] über psychische Folgen wie Belastungen, Depressionen sowie die Vereinsamung von Kindern berichtet. Das folgende Zitat eines sechsjährigen Mädchens stammt aus der Studie „Kind sein in Zeiten von Corona“ (Langmeyer et al. 2020)[2] des Deutschen Jugendinstituts (DJI) zu den Veränderungen in der Pandemie: „Also ich kann nicht mehr normal zur Schule gehen, ich habe jetzt nur noch einen Tag, ich würde öfters gehen wollen, und ich würde sehr gerne mehr mit anderen zusammen sein. Das hat mir Corona so geklaut.“ (Ebd.: 95)

In der Coronakrise wird die Situation von Kindern weitgehend auf dominante Diskurse wie Bildung, psychosoziale Risiken und Gewalt reduziert. Dass Kinder insbesondere in Zeiten einer globalen Krise, die mit einer Zunahme von häuslicher Gewalt, Anspannung und Druck bei der Betreuung und Beschulung einhergeht, geschützt und gestärkt werden müssen, ist fundamental wichtig. Gleichzeitig sollte der Blick auf ihre Bedürfnisse und Belange jenseits dieser vorherrschenden Themenbereiche nicht außer Acht geraten. Es geht zudem um ihre Perspektiven auf die Krise und die getroffenen Maßnahmen, um ihre sozialräumlichen Bezüge vor dem Hintergrund der Kontaktbeschränkungen, um Zugänge und vor allem um die Frage danach, inwieweit sie in das Krisenmanagement und in wissenschaftliche Forschungen als Akteur_innen einbezogen werden.

In diesem Beitrag analysiere ich, welche Bilder von Kindern im wissenschaftlichen Diskurs seit Beginn der Pandemie hervorgebracht wurden. Anhand der Studien zur Situation von Kindern in der Coronakrise - der „COPSY“-Studie (2020/2021), der „KiCo“-Studie (2020) und der Studie des 
DJI „Kind sein in Zeiten von Corona“ (2020) - untersuche ich, inwiefern Räume von Kindern (institutionelle Räume, Wohnräume, Freizeiträume, öffentliche Räume, urbane Räume) beachtet oder auch ausgeblendet wurden. Besonders berücksichtige ich die Auswirkungen sozialer Ungleichheiten auf die Lebenslagen von Kindern in der Krise und decke Stigmatisierungen auf, die in Zusammenhang mit sogenannten benachteiligten Lebenslagen reproduziert und produziert werden. Mit Blick auf die (mangelnde) Beteiligung von Kindern in der Forschung greife ich auf, welche Stimme Kindern in den Studien zugesprochen wurde und inwiefern sie in die Forschung einbezogen wurden - oder auch nicht.

Ausgehend von dem wissenschaftlichen Diskurs über Kinder in der Coronakrise blicke ich auf den Beitrag der Stadtforschung in Deutschland zu diesem Thema. Warum hat sie sich der Situation von Kindern in der Coronakrise bislang - bis auf wenige Ausnahmen wie beispielsweise dieses Themenheft kaum angenommen? Können doch besonders mithilfe der Ansätze und Perspektiven einer kritischen Stadtforschung die bisher in der Coronakrise wenig beachteten Bedarfe, Räume, Alltagsbezüge, Bilder und Wissensbestände von Kindern - jenseits dominierender Diskurse über Bildung, Gewalt, Kinderbetreuung - differenziert herausgearbeitet werden. Dabei können Fragen nach Machtstrukturen, nach sozialen Ungleichheiten in Verbindung mit Geschlecht, Migration und sozialer Klasse sowie nach (mangelnder) Handlungsmacht von Kindern aus einer raumanalytischen und kritischen Perspektive betrachtet und in den wissenschaftlichen Diskurs gebracht werden.

\section{Räume von Kindern in der Coronakrise}

\section{Kindheiten im institutionellen Gefüge von Kita und Schule}

Mit Blick auf die Frage nach Öffnungs- und Schließungsmaßnahmen von institutionellen Räumen der Kinder wie Kindertagesstätten und Schulen drehen sich die Diskurse und wissenschaftlichen Untersuchungen um Fragen nach der Übertragung des Virus durch Kinder und den Auswirkungen auf die Organisation der Institutionen. Aus medizinischer und sozialwissenschaftlicher Perspektive untersucht beispielsweise die „Corona-KiTaStudie“[3] des Robert-Koch-Instituts (RKI) und des DJI seit Mai 2020 die Praxis der Schließungen beziehungsweise Öffnungen der Einrichtungen im Zeitverlauf, die Organisations- und Personalstrukturen sowie den Umgang mit Infektionsfällen in Kindertagesstätten. Selbstverständlich sind dies wichtige Faktoren für die Planung und Organisation der Kindertagesbetreuung und der Kitas, die mit der Betreuung von Kindern im Allgemeinen und der Ausübung der Erwerbsarbeit der Eltern eng zusammenhängen. Kinder werden allerdings verstärkt in Bezug auf ihre Ansteckungsfähigkeit betrachtet, die mit der institutionellen Gestaltung der Betreuung einhergeht - wie beispielsweise in Bezug auf Entscheidungen über die Schließung oder die Öffnung von Kitas sowie die Organisation der Notbetreuung. Wie wirkt sich nun die veränderte Betreuungssituation auf die Räume der Kinder im Alltag aus? Welche Perspektive haben sie selbst auf die sich permanent ändernde Situation durch Schließungen und Öffnungen von Kitas, Notbetreuungsangebote, die Betreuung zu Hause oder anderswo? 
Seit dem Beginn der Pandemie wurden die Auswirkungen von Kita- und Schulschließungen auf Bildungschancen und Zukunftsperspektiven junger Menschen öffentlich und wissenschaftlich debattiert. Anknüpfend an den institutionellen Raum der Kita thematisierten wissenschaftliche Studien die Erfahrungen und Auswirkungen des Homeschooling, die berechtigte Sorge um (fehlende) Anschlüsse an das nächste Schuljahr, bedrohte Bildungsabschlüsse und die Gestaltung von Schule in der Coronazeit mit Wechselund Distanzunterricht (Fickermann/Edelstein 2020; Andresen et. al 2020; Bertelsmann Stiftung 2020; Langmeyer et al. 2020). Ohne Frage ist die Auseinandersetzung mit den Folgen der Krise bezüglich Bildungschancen, einer drohenden Zunahme von Bildungsungleichheiten und fehlender Teilhabe an Bildung grundlegend.[4] Der Fokus auf Bildung reduziert Kinder gleichzeitig auf den institutionellen Raum von Kita und Schule.

Angesichts der beschriebenen Einengung lege ich in diesem Beitrag den Schwerpunkt auf Räume jenseits dieser Bereiche - den Raum der Familie, den Wohnraum, den Freizeitraum, den öffentlichen Raum und schließlich den (missachteten oder vergessenen) urbanen Raum von Kindern in der Stadt.

\section{Kindheiten im Raum der Familie}

Die Auseinandersetzung mit der sozialen Position von Kindern in der Familie, die Perspektive auf Kinder im Familiensystem beziehungsweise die Einbeziehung der Eltern in wissenschaftliche Studien stehen im Zusammenhang mit dem jungen Alter und der (nun noch größeren) Abhängigkeit der Kinder von ihren Eltern. So geht es in den hier betrachteten Studien zu einem großen Teil um die Familien und ihre Kinder, wo seit Beginn der Krise verstärkt die Kinderbetreuung und das Homeschooling stattfinden. Welche Familien wurden in den Studien überhaupt in den Blick genommen? Welche Familienbilder werden gezeichnet? Welche Familien wurden nicht berücksichtigt?

Die bundesweite „KiCo“-Studie (Andresen et al. 2020), die im Forschungsverbund „Kindheit - Jugend - Familie in der Corona-Zeit“[5] entstanden ist und im Mai 2020 veröffentlicht wurde, arbeitet die Auswirkungen der Krise auf die Familien (familiärer Alltag und Atmosphäre, Vereinbarkeit der Betreuung mit Erwerbsarbeit), die Herausforderungen und die soziale Position von Kindern und deren Aushandlung in der Institution Familie umfassend heraus. Dabei wird die Situation von Kindern im Kontext Familie betrachtet. Dies spiegelt sich im Forschungsdesign der Studie wider, indem die Sichtweisen von Kindern berücksichtigt werden, allerdings nur ansatzweise und stellvertretend über deren Eltern. Dazu wurden Familien mit Kindern unter 15 Jahren anhand eines Onlinefragebogens befragt. Die Eltern wurden gebeten, ,sich entweder in jedes einzelne ihrer Kinder hineinzuversetzen oder den Fragebogen mit ihren Kindern gemeinsam auszufüllen“ (ebd.: 6). Die Perspektiven der befragten Kinder können so nur über den Umweg der Eltern rekonstruiert werden. Damit werden Kinder kaum als Subjekte wahrgenommen, die eigenständig ihre Sichtweise vertreten. Mit Blick auf die Beteiligung von Kindern in der Forschung stellt sich die Frage: Ist diese Vorgehensweise geeignet, um die Perspektiven der Kinder adäquat wiederzugeben?[6] 
Die „KiCo“-Studie arbeitet die heterogenen Gestaltungsweisen des Familienalltags in der Krise heraus, wobei Familie als „Seismograph“(ebd.: 23) für die gesellschaftlichen Problemlagen identifiziert und die These vertreten wird, dass ,sich in Familien die sozialen Folgen der Regulation der Pandemie bündeln"(ebd.). Selbstkritisch wird angemerkt, dass sozioökonomisch schlechter gestellte Familien weniger erfasst worden seien, da es schwieriger sei, diese zu erreichen (ebd.: 7). Wie können in zukünftigen wissenschaftlichen Untersuchungen auch diese Lebenslagen berücksichtigt und ökonomisch schlechter gestellte Familien besser erreicht werden?

Auch in der DJI-Studie sind sogenannte benachteiligte Familien unterrepräsentiert. Die an der Onlinebefragung und der Interviewstudie beteiligten Familien waren laut der Forscher_innen finanziell recht gut ausgestattet und verfügten über höhere Bildungsabschlüsse (Langmeyer et al. 2020: $10 \mathrm{ff}$.). Kritisch zu hinterfragen sind außerdem die Familienbilder, die mit der Studie (re-)produziert werden. Auch hier zeigt sich eine frappierende Lücke, da heterogene Familienkonstellationen (Regenbogen-, Trennungs- und Patchworkfamilien) gegenüber heteronormativen Familien unterrepräsentiert sind.

Im qualitativen Teil der DJI-Studie (der ergänzend zur Onlinebefragung durchgeführt wurde) kamen Kinder zwischen 6 und 14 Jahren mittels telefonischer Interviews zu Wort. Ältere Kinder führten diese Interviews allein; bei jüngeren Kindern blieb ein Elternteil in der Nähe (ebd.: 73). In Pandemiezeiten ist die Durchführung von Interviews mit Kindern natürlich schwieriger, und insofern ist positiv, dass überhaupt telefonische Interviews durchgeführt wurden. Um jedoch insbesondere jüngeren Kindern eine authentische Stimme im wissenschaftlichen Diskurs geben zu können, sind altersgerechte methodische Verfahren, die durchaus arbeits- und zeitintensiver sind, wichtig.

Letzteres lässt sich in quantitativen Studien noch schwerer umsetzen, wie in der „COPSY“-Studie erkennbar wird: Direkt befragt wurden hier 11- bis 17-Jährige, wohingegen bei den 7- bis 10-Jährigen die Antworten von den Eltern eingetragen wurden. Auch hier - in quantitativen Befragungen - sollte allen Kindern die Möglichkeit gegeben werden, die Fragen selbstständig zu beantworten und damit als Akteur_innen und Expert_innen ihrer eigenen Alltagswelt zu agieren. Insbesondere dann, wenn der Themenschwerpunkt der Studie die direkte Alltagswelt von Kindern - auch jüngeren - betrifft.

Die „COPSY“-Studie hat die Auswirkungen der Coronakrise auf das Wohlbefinden und die psychische Gesundheit von Kindern und Jugendlichen untersucht. Es wird eine verschlechterte psychische Situation konstatiert, die unter anderem mit zunehmenden Konflikten und mit Stress in den Familien in Zusammenhang gebracht wird (Ravens-Sieberer et al. 2021, 2020: 828). Jedoch wird hier stark zwischen Kindern aus privilegierten und Kindern aus benachteiligten Familien differenziert. Die Studie zielt zwar darauf ab, auf die durch die Krise hervorgerufene verschlechterte Situation von Kindern aus benachteiligten Familien aufmerksam zu machen. Dies ist insbesondere mit Blick auf die Zunahme sozialer Ungleichheiten in der Krise wichtig. Im Ergebnisteil der Studie wird jedoch ein reduzierendes und stigmatisierendes Bild von sozioökonomisch schlechter gestellten Familien mit Migrationshintergrund gezeichnet: Risikofaktoren werden in der 
Migrationsgeschichte, in geringen Einkommen und niedrigen Bildungsstatus der Familien ausgemacht (Ravens-Sieberer et al. 2020: 829). Eine solche verengte Perspektive wird der heterogenen Lebenssituation sogenannter benachteiligter Kinder und ihrer Familien nicht gerecht. Die Analyse müsste differenzierter auf den jeweiligen situativen Kontext gerichtet sein. So führt etwa ein niedriger Bildungsstatus nicht automatisch dazu, dass Eltern ihre Kinder in Bezug auf Bildung nicht unterstützen (können). Anstatt Migrationshintergründe zu problematisieren, sollte beispielsweise die Mehrsprachigkeit der Kinder und ihrer Familien als Ressource betrachtet werden. Es geht darum, den vorstrukturierten Blick sowie die Kategorien, die in den wissenschaftlichen Untersuchungen herausgebildet werden, zu hinterfragen.

Heterogene Familienbilder und -arrangements sind in den hier genannten Studien wenig beachtet worden. Um der Diversität der Familienkonstellationen und Betreuungsarrangements, in denen Kinder aufwachsen, mehr Anerkennung zukommen zu lassen, ist es wichtig, dass diese in den Forschungsdesigns und der methodischen Konzeption mehr Beachtung finden und darin sichtbar werden. Kritische Auseinandersetzungen mit Kinderarmut und den prekären Lebenslagen von Familien in der Krise finden sich in den Studien von Gerda Holz und Antje Richter-Kornweitz (2020) sowie von Michael Klundt und Norbert Müller (2020).

\section{(Marginalisierte) Wohnräume}

Seit Beginn der Pandemie verbringen wir alle mehr Zeit in den ,eigenen vier Wänden" - auch die Kinder. Wie viel Platz einem Kind zur Verfügung steht, ist sehr heterogen und variiert nach sozioökonomischem Hintergrund der Eltern sowie nach Wohnform. Zudem gestaltet sich das Wohnen je nach Stadt, Stadtteil und Region sehr unterschiedlich. Diese Ungleichheiten treten aktuell mit den Debatten über hohe Inzidenzzahlen in "sozialen Brennpunkten“ hervor, wobei ein direkter Zusammenhang zwischen hoher Inzidenz und beengtem Wohnraum konstatiert wird (Süddeutsche Zeitung 2021). Vom Wohnraum und der materiellen Ausstattung hängt in der Coronakrise ab, wie gut der Alltag bewältigt werden kann und inwieweit man sich überhaupt vor dem Virus schützen kann. Privilegien wie auch Benachteiligungen lassen sich entlang der Frage nach der Wohnlage, der Wohnform, der Größe der Wohnfläche, der Verfügbarkeit privater Außenflächen, der Qualität der Bausubstanz, der technischen Ausstattung oder dem Zustand der sanitären Anlagen ausmachen.

Auch die Studien zur Situation von Kindern in der Coronakrise nehmen Bezug auf den Wohnkontext. Beispielsweise werden in der BertelsmannStudie fehlende Rückzugsräume in Wohnungen mit negativen Auswirkungen auf das Homeschooling in Zusammenhang gebracht (Bertelsmann 2020: 9). Die „COPSY“-Studie benennt als Ursachen für eine Verschlechterung der psychischen Situation von Kindern unter anderem mangelnde Rückzugsräume und beengten Wohnraum - neben niedrigen Bildungsabschlüssen, einem Migrationshintergrund der Eltern und einem "schlechten Familienklima“ (Ravens-Sieberer et al. 2020: 829). Wie bereits ausgeführt, treten hier jedoch stigmatisierende Betrachtungsweisen in Verknüpfung mit klassistischen und rassistischen Diskursen in Erscheinung. 
Ein etwas anderes Bild zeichnet die „KiCo“-Studie, die Unterschiede zwischen den Rückzugsräumen von Kindern und Erwachsenen beschreibt. Demnach verfügten 90 Prozent der Kinder über einen ungestörten Raum, während über 40 Prozent der Erwachsenen angeben, keinen solchen Raum zu haben (Andresen et al. 2020: 20). Wie bereits ausgeführt, ist zu berücksichtigen, dass an der „KiCo“-Studie größtenteils gut situierte Familien teilgenommen haben.

Auch die DJI-Studie greift die Wohnsituation auf: Der überwiegende Teil der hier befragten Familien (8o Prozent) verfügt über ein Kinderzimmer. 62 Prozent der Familien sind mit eigenem Kinderzimmer, Garten oder Terrasse ausgestattet. Demgegenüber haben neun Prozent der Familien weder für jedes Kind ein eigenes Zimmer noch Garten oder Terrasse (Langmeyer et al. 2020: 26). Die recht gute Wohnsituation der befragten Familien spiegelt (auch hier) relativ privilegierte Lebenslagen wider.

Betrachtet man die Erkenntnisse hinsichtlich der Auswirkungen von Wohnformen und Wohnräumen auf den Alltag von Kindern in der Krise, bleiben noch einige Fragen offen: Hängen Rückzugs- und Freiräume von Kindern ausschließlich mit dem Wohnraum zusammen? Welche weiteren Faktoren spielen eine Rolle? Welchen Einfluss hat der urbane Kontext, in den der Wohnraum eingebettet ist (Stadtteil, Wohngebiet, Region)? Insbesondere die kritische Stadtforschung kann hierzu - mit Blick auf langfristige Auswirkungen der Coronakrise und soziale wie räumliche Ungleichheiten in der Stadt einen differenzierten, (macht-)kritischen Beitrag leisten. Beispielsweise kann aus stadtsoziologischer, stadtethnographischer, stadtplanerischer und/oder stadtgeographischer Perspektive interdisziplinär über zukünftige Wohnformen in der Stadt nachgedacht werden. Es können konzeptionelle Ansätze (weiter-)entwickelt werden, in denen vor allem die Bedarfe von Kindern Berücksichtigung finden. Neben Zugängen zu Grünflächen, zum öffentlichen Nahverkehr sowie zu einer kindgerechten Infrastruktur ist die Nähe zu Bildungsinstitutionen, Sport- und Freizeitflächen wichtig.

\section{Freizeiträume - öffentliche Räume - urbane Räume?}

In den Studien zur Situation von Kindern in der Coronakrise kommen Fragen nach der (eingeschränkten) Nutzung und Aneignung von Orten, Plätzen und Räumen im öffentlichen Raum kaum bis gar nicht vor. Nur vereinzelt spielt das Freizeitverhalten eine Rolle, wobei Bezüge zum Wohnraum, zu Plätzen und Orten im öffentlichen Raum (etwa Spielplätzen) marginal hergestellt werden. Veränderte Freizeitroutinen greift etwa das DJI auf, wobei nach Kindergartenund Schulalter differenziert wird. Insgesamt wird - wenig überraschend - herausgearbeitet, dass Kinder in der Coronakrise mehr Zeit zu Hause verbringen. In der Studie werden unterschiedliche Wohnverhältnisse anhand des Vorhandenseins privater Freiflächen (Garten, Terrasse, Balkon) untersucht. So beobachten 42 Prozent der Eltern mit privaten Freiflächen einen Anstieg kindlicher Aktivitäten draußen - im Vergleich zu 23 Prozent bei den Eltern, die nicht über Garten oder Terrasse verfügen (Langmeyer et al. 2020: 36).

Die beschriebenen quantitativen Untersuchungen bleiben jedoch an der Oberfläche. Um das Freizeitverhalten von Kindern in der Coronakrise differenziert beschreiben zu können, braucht es tiefergehende Analysen. 
Beispielsweise bleibt die Frage der Erreichbarkeit von Orten wie Parks, Spielplätzen oder Straßen in der Stadt, die von Kindern genutzt und angeeignet werden können, in diesen Studien offen.

Ebenso verkürzt - und möglicherweise den methodischen Grenzen von quantitativen Befragungen geschuldet - werden darüber hinaus regionale Unterschiede angeschnitten, und zwar zwischen Kindern in der Stadt und Kindern auf dem Land (ebd.). In recht knapper Darstellung wird die These untermauert, Kinder im ländlichen Raum verbrächten im Vergleich zu Stadtkindern mehr Zeit draußen (ebd.). Das Ergebnis kann jedoch nicht befriedigen - es müsste genauer nach privilegierten und marginalisierten Wohnlagen und -räumen, nach Stadt und Region, räumlicher Ausstattung der direkten Wohnlage differenziert werden. Es bedarf detaillierterer Fragestellungen, die sich qualitativer oder ethnographischer Forschungsmethoden bedienen, um vielfältiges Wissen über die Unterschiede im Alltag von Kindern auf dem Land und in der Stadt zu erhalten. So könnte beispielsweise genauer analysiert werden, wie Kinder das „Rumhängen“ (ebd.), das Eltern in der Stadt bei ihren Kindern stärker wahrnehmen als Eltern auf dem Land, ausfüllen und deuten.

\section{Kinder in der Stadt in der Coronakrise: Wo bleibt denn nun der urbane Raum?}

Urbane Räume stellen für Kinder insbesondere in der Coronakrise wichtige Orte für Bewegung und Kontakte dar. In den von mir analysierten Studien werden urbane Räume - bis auf Querbezüge zu Freizeit und Wohnen - jedoch kaum bis gar nicht berücksichtigt. Es fehlen somit Forschungsarbeiten und wissenschaftliche Analysen beispielsweise zur veränderten Nutzung von Plätzen und Orten in der Stadt oder zur Aneignung von städtischen Räumen durch Kinder (vgl. Kogler 2018).

In Abbildung 1 ist zu sehen, auf welche Art und Weise Kinder ihre Perspektive und Gefühlslage - hier in Köln-Zollstock - zum Ausdruck bringen. Solche Statements zur Krise in Form von Zeichnungen, Sprechblasen und Kommentierungen, oft mit Kreide gezeichnet, ließen sich auf den Straßen vieler Städte ausmachen. Sie verdeutlichen neben Aneignungen des städtischen Raums

Abb. 1 Kinder in Köln-Zollstock (Eigene Aufnahme, 2020)

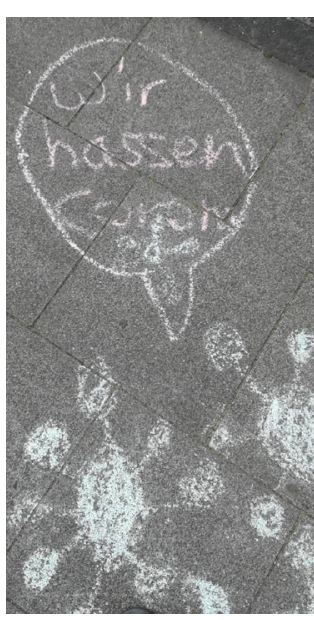
die Positionierungen von Kindern zur Coronakrise sowie Taktiken, Spuren zu hinterlassen und sich im städtischen Raum zu platzieren.

Solche und weitere Fragestellungen zu Räumen von Kindern in der Stadt - als Möglichkeitsräume, Rückzugsräume oder Nischen - bieten interessante Forschungsfelder in und nach der Coronakrise - insbesondere für eine sich als kritisch verstehende Stadtforschung.

\section{Für mehr Räume von Kindern in der Stadtforschung}

Für eine kritische Stadtforschung besteht die Chance und gleichzeitig die Notwendigkeit, sich mit Fragen der Repräsentation von Kindern in der Stadt, der Aneignung von Räumen durch Kinder und den Beteiligungsmöglichkeiten und -formaten in Städten und Regionen auseinanderzusetzen. Kindheiten und Kinderleben in der Stadt sind in der Stadtforschung nach wie vor unterrepräsentiert. Besondere Berücksichtigung sollten städtische 
Exklusionsprozesse finden - insbesondere mit Blick auf die Situation von Kindern in marginalisierten Regionen und Städten. Auf der Grundlage der Betrachtung sozialer Ungleichheiten und Machtasymmetrien muss nach Handlungsmöglichkeiten, Teilhabechancen und der Stärkung der Mitbestimmung von Kindern - auch in der Forschung - gefragt werden. Aufbauend auf bestehende Studien und Publikationen zu Kindern in der Stadt beziehungsweise Kindheit und Raum (exemplarisch Bollig/Alberth/ Schindler 2020; Kraftl 2020; Holloway/Valentine 2000; Braches-Chyrek/ Röhner 2016; Reutlinger/Brüschweiler 2016) kann die kritische Stadtforschung wertvolle Impulse zur bislang unterrepräsentierten Thematik „Kinder in der Stadt" geben, in diesem Forschungsfeld Wissen generieren und sich in wissenschaftliche sowie öffentliche und politische Debatten einbringen.

Besonders angesichts des Andauerns der Coronakrise, die bleibende Auswirkungen und gravierende Folgen für die (zukünftigen) Lebenslagen von Kindern haben wird, sind Studien unter der Beteiligung von Kindern wichtig und sinnvoll, um ihre Sichtweisen in die wissenschaftliche Auseinandersetzung und Reflexion einzubinden (Schreiber 2017). Die Kindheitsforschung hat hierzu bereits unterschiedliche methodologische und methodische Anreize geliefert (Hartnack 2019; Heinzel 2000). Zur Wahrnehmung des Stadtraums durch Kinder bieten Methoden der Stadtforschung wichtige Ausgangspunkte - beispielsweise aus der ethnographischen, visuellen Stadtforschung die Fotospaziergang-Interviews, Itinéraire [7] (Cuny 2020), verschiedene Mapping-Ansätze zu Orten und Räumen im Quartier, im Stadtteil oder in der Stadt sowie urbane Soundscapes. Zur Stärkung der Teilhabe von Kindern in der Forschung können kreative und kindgerechte Methoden gemeinsam mit Kindern entwickelt, ausprobiert und angewendet werden. Inspirationen hierzu kann auch die partizipative Forschung liefern (von Unger 2014).

\section{Endnoten}

[1] Bei der Folgestudie wurden im Zeitraum von Mitte Dezember 2020 bis Mitte Januar 2021 über 1.00o Kinder und Jugendliche sowie mehr als 1.60o Eltern online befragt. Themen waren die Coronakrise, Schule, Freund_innen, Familie sowie psychische Folgen und Probleme. Dieser Studie ging eine erste Befragung im Juni 2020 voraus (80 Prozent der hier befragten Kinder und Eltern nahmen an der Folgebefragung teil). Für Sommer 2021 ist eine weitere Befragung anvisiert (Ravens-Sieberer et al. 2021, 2020).

[2] Im Zeitraum vom 22. April bis zum 21. Mai 2020 wurde eine Onlinebefragung der Eltern durchgeführt, an der insgesamt 12.629 Personen teilgenommen haben. Ergänzend wurde im Zeitraum 26. Mai bis 8. Juni 2020 eine Interviewstudie (21 Interviews) durchgeführt. Beteiligt waren jeweils ein Elternteil und ein Kind zwischen 6 und 14 Jahren (Langmeyer et al. 2020: $8 \mathrm{ff} ., 71 \mathrm{ff}$.).

[3] Die „Corona-KiTa-Studie“ wird vom RKI gemeinsam mit dem DJI durchgeführt. In der Längsschnittstudie werden die Herausforderungen der Kindertagesbetreuung und die Rolle von Kindern bei der Übertragung des Virus untersucht. Analysiert wird die schrittweise Öffnung von Kindertageseinrichtungen, wozu verschiedene Datenquellen herangezogen werden (Befragungen der Einrichtungen, der Fachkräfte, der Eltern und Daten zu Covid-19-Fällen). Ein Bericht zu den Ergebnissen wird monatlich und pro Quartal veröffentlicht (DJI/RKI 2021).

[4] Eine interessante Fragestellung für weitere Forschungen, die sich mit dem Digitalisierungsschub seit Beginn der Pandemie stellt, lautet: Welche neuen virtuellen (Bildungs-) 
Räume werden in der Coronakrise hervorgebracht und wie eignen Kinder sich diese an? (Vgl. hierzu ansatzweise Langmeyer et al. 2020: 97 f.)

[5] Der Forschungsverbund „Kindheit - Jugend - Familie in der Corona-Zeit“ setzt sich zusammen aus dem Institut für Sozial- und Organisationspädagogik der Stiftung Universität Hildesheim und dem Institut für Sozialpädagogik und Erwachsenenbildung der Universität Frankfurt am Main in Kooperation mit der Universität Bielefeld (Stiftung Universität Hildesheim 2021).

[6] In Bezug auf die Aussagen über Kinder muss nach möglichen Verzerrungen gefragt werden. Bestimmte Themen wie Konflikte innerhalb der Familie, Probleme mit Eltern oder Geschwistern können beispielsweise gar nicht erfasst werden.

[7] Das Fotospaziergang-Interview geht auf Jean-Yves Petiteau zurück, hier wird ein „im Gehen stattfindendes Interview fotografisch festgehalten und als eine teils sehr poetische Komposition aus Text und Bild vorgetragen oder publiziert" (Cuny/Färber/ Preissing 2019: 56 f.).

\section{Autor_innen}

Sonja Preissings Forschungsschwerpunkte sind Jugend-, Stadt- und Migrationsforschung sowie Qualitative Forschungsmethoden.

sonja.preissing@iu.org

\section{Literatur}

Andresen, Sabine / Lips, Anna / Möller, Renate / Rusack, Tanja / Schröer, Wolfgang / Thomas, Severine / Wilmers, Johanna (2020): Kinder, Eltern und ihre Erfahrungen während der Corona-Pandemie. Erste Ergebnisse der bundesweiten Studie KiCo. Hildesheim: Universitätsverlag Hildesheim. https:/hildok.bsz-bw.de/frontdoor/index/ index/docId/1081 (letzter Zugriff am 1.5.2021).

Bertelsmann Stiftung (2020): Factsheet. Kinderarmut in Deutschland. https://www. bertelsmann-stiftung.de/de/publikationen/publikation/did/factsheet-kinderarmut-indeutschland (letzter Zugriff am 1.5.2021).

Bollig, Sabine / Alberth, Lars / Schindler, Larissa (Hg.) (2020): Materialitäten der Kindheit. Körper - Dinge - Räume. Wiesbaden: VS Verlag für Sozialwissenschaften.

Braches-Chyrek, Rita / Röhner, Charlotte (Hg.) (2016): Kindheit und Raum. Opladen: Verlag Barbara Budrich.

Cuny, Cécile (2020): Itinéraire d'une collaboration. Entretien avec Bernard Renoux. In: Cécile Cuny / Alexa Färber / Anne Jarrigeon (Hg.), L'Urbain par l'image. Collaborations entre arts visuels et sciences sociales. Grane: Créaphis Editions, 94-103.

Cuny, Cécile / Färber, Alexa / Preissing, Sonja (2019): Autonomie und Zusammenarbeit. Zur zeitlichen Dimensionierung kooperativer Fotografie- und Stadtforschung. In: Stefan Groth / Christian Ritter (Hg.), Zusammen arbeiten. Praktiken der Koordination und Kooperation in kollaborativen Prozessen. Bielefeld: transcript, 51-77.

DJI/RKI - Deutsches Jugendinstitut / Robert Koch-Institut (2021): Corona-KiTa-Studie. https://corona-kita-studie.de/ (letzter Zugriff am 2.5.2021).

Fickermann, Detlef / Edelstein, Benjamin (Hg.) (2020): „Langsam vermisse ich die Schule ..... Schule während und nach der Corona-Pandemie. Beiheft 16 der Zeitschrift für Erziehungswissenschaften, Bildungspolitik und pädagogische Praxis. Münster/New York: Waxmann Verlag.

Fischer, Sabine / Rahn, Peter (Hg.) (2017): Kind sein in der Stadt. Bildung und ein gutes Leben. Opladen: Verlag Barbara Budrich.

Hartnack, Florian (Hg.) (2019): Qualitative Forschung mit Kindern. Herausforderungen, Methoden und Konzepte. Wiesbaden: VS Verlag für Sozialwissenschaften.

Heinzel, Friederike (Hg.) (2000): Methoden der Kindheitsforschung. Ein Überblick über Forschungszugänge zur kindlichen Perspektive. Weinheim/München: Juventa Verlag.

Holloway, Sarah / Valentine, Gill (2000): Spatiality and the New Social Studies of Childhood. In: Sociology 34/4, 763-783. 
Holz, Gerda / Richter-Kornweitz, Antje (2020): Corona-Chronik. Gruppenbild ohne (arme) Kinder. Eine Streitschrift. https://www.iss-ffm.de/fileadmin/assets/themenbereiche/ downloads/Corona-Chronik_Streitschrift_final.pdf (letzter Zugriff am 1.5.2021).

Klundt, Michael / Müller, Norbert (2020): Krisengerechte Kinder statt kindergerechtem Krisenmanagement? Auswirkungen der Corona-Krise auf die Lebensbedingungen junger Menschen. Studie für die Bundestagsfraktion DIE LINKE. https://www.linksfraktion.de/ publikationen/detail/krisengerechte-kinder-statt-kindergerechtem-krisenmanagement/ (letzter Zugriff am 1.5.2021).

Kogler, Raphaela (2018): Kinderräume erkunden. Partizipative Stadtforschung und -planung mit Kindern. In: Informationen zur Raumentwicklung 2/2018, 40-51.

Kraftl, Peter (2020): After childhood. Re-thinking environment, materiality and media in children's lives. London: Routledge.

Langmeyer, Alexandra / Guglhör-Rudan, Angelika / Naab, Thorsten / Ulen, Marc / Winklhofer, Ursula (2020): Kind sein in Zeiten von Corona. Ergebnisbericht zur Situation von Kindern während des Lockdowns im Frühjahr 2020. https://www.dji.de/fileadmin/ user_upload/dasdji/news/2020/DJI_Kindsein_Corona_Ergebnisbericht_2020.pdf (letzter Zugriff am 1.5.2021).

Ravens-Sieberer, Ulrike / Kaman, Anne / Otto, Christiane / Adedeji, Adenkule / Devine, Janine / Erhart, Michael / Napp, Ann-Kathrin / Becker, Marcia / Blanck-Stellmacher, Ulrike / Löffler, Constanze / Schlack, Robert / Hurrelmann, Klaus (2020): Psychische Gesundheit und Lebensqualität von Kindern und Jugendlichen während der COVID19-Pandemie. Ergebnisse der COPSY-Studie. In: Deutsches Ärzteblatt 117/48, 828-829. https://cdn.aerzteblatt.de/pdf/117/48/m828.pdf?ts=05\%2E11\%2E2020+14\%3Ao9\% 3A32 (letzter Zugriff am 1.5.2021).

Ravens-Sieberer, Ulrike / Kaman, Anne / Erhart, Michael / Devine, Janine / Schlack, Robert / Otto, Christina (2021): Impact of the COVID-19 pandemic on quality of life and mental health in children and adolescents in Germany. In: European Child \&Adolescent Psychiatry, 25.1.2021. https://edoc.rki.de/bitstream/handle/176904/7728/Ravens-Sieberer2021_ Article_ImpactOfTheCOVID-19PandemicOnQ.pdf?sequence=1\&isAllowed=y (letzter Zugriff am 1.5.2021).

Reutlinger, Christian / Brüschweiler, Bettina (2016). Sozialgeographien der Kinder. Eine Spurensuche in mehrdeutigem, offenem Gelände. In: Rita Braches-Chyrek / Charlotte Röhner (Hg.), Kindheit und Raum. Opladen: Barbara Budrich, 37-64.

Schreiber, Verena (2017): Forschen mit Kindern. In: Quasus. Qualitatives Methodenportal zur Qualitativen Sozial-, Unterrichts- und Schulforschung. https:/quasussite.wordpress. com/forschen-mit-kindern/ (letzter Zugriff am 12.3.2021).

Stiftung Universität Hildesheim (2021): JuCo und KiCo. Befragungen von jungen Menschen und Eltern während der Corona-Pandemie. https://www.uni-hildesheim.de/ fb1/institute/institut-fuer-sozial-und-organisationspaedagogik/forschung/laufendeprojekte/juco-und-kico/ (letzter Zugriff am 2.5.2021).

Süddeutsche Zeitung (2021): Köln: Ab Montag Impfungen in sozialen Brennpunkten starten. In: Süddeutsche Zeitung, 30.4.2021. https://www.sueddeutsche.de/gesundheit/ gesundheit-koeln-koeln-ab-montag-impfungen-in-sozialen-brennpunkten-starten-dpa. urn-newsml-dpa-com-20090101-210430-99-417893 (letzter Zugriff am 1.5.2021).

von Unger, Hella (2014): Partizipative Forschung. Einführung in die Forschungspraxis. Wiesbaden: VS Verlag für Sozialwissenschaften. 